TRANSACTIONS OF THE

AMERICAN MATHEMATICAL SOCIETY

Volume 231, Number 2, 1977

\title{
CYCLIC PURITY VERSUS PURITY IN EXCELLENT NOETHERIAN RINGS
}

BY

\author{
MELVIN HOCHSTER( ${ }^{(1)}$
}

\begin{abstract}
A characterization is given of those Noetherian rings $R$ such that whenever $R$ is ideally closed ( $\equiv$ cyclically pure) in an extension algebra $S$, then $R$ is pure in $S$. In fact, $R$ has this property if and only if the completion $(A, m)$ of each local ring of $R$ at a maximal ideal has the following two equivalent properties:

(i) For each integer $N>0$ there is an m-primary irreducible ideal $I_{N} \subset m^{N}$.

(ii) Either $\operatorname{dim} A=0$ and $A$ is Gorenstein or else depth $A \geqslant 1$ and there is no $P \in \operatorname{Ass}(A)$ such that $\operatorname{dim}(A / P)=1$ and $(A / P) \oplus(A / P)$ is embeddable in $A$.

It is then shown that if $R$ is a locally excellent Noetherian ring such that either $R$ is reduced (or, more generally, such that $R$ is generically Gorenstein), or such that Ass $(R)$ contains no primes of coheight $\leqslant 1$ in a maximal ideal, and $R$ is ideally closed in $S$, then $R$ is pure in $S$. Matlis duality and the theory of canonical modules are utilized. Module-theoretic analogues of condition (i) above are, of necessity, also analyzed.

Numerous related questions are studied. In the non-Noetherian case, an example is given of a ring extension $R \rightarrow S$ such that $R$ is pure in $S$ but $R[[T]]$ is not even cyclically pure in $S[[T]]$.
\end{abstract}

0 . Introduction. All rings are commutative, with identity, and modules are unital. Recall that a submodule $N$ of a module $M$ (or the map $N \rightarrow M$ ) over a ring $R$ is called pure (respectively, cyclically pure) if for every $R$-module (respectively, every cyclic $R$-module) $E$ the map $N \otimes E \rightarrow M \otimes E$ is injective. A case of particular interest is the one where $N=R$ and $E=S$ is an extension algebra of $R$ : in this case the cyclic purity of $R$ in $S$ asserts that for every ideal $I$ of $R, I S \cap R=I$ (let $E=R / I$ in the definition of cyclic purity). This condition is sometimes expressed by the phrase " $R$ is ideally closed in $S$ ". Quite generally, if $M / N$ is finitely presented, then $N$ is pure in $M$ if and only if $N$ is a direct summand of $M$. We refer the reader to [3], [14], [15], [20, p. 64], and [24] for basic facts about purity.

Our original objective was to give a really useful condition on a Noetherian

Received by the editors February 23, 1976.

AMS (MOS) subject classifications (1970). Primary 13E05, 13H10, 13B99, $13 \mathrm{C} 99$.

(1) The author was supported, in part, by a grant from the National Science Foundation.

- American Mathematical Society 1977 
ring $R$ such that whenever $R$ satisfies the condition and is cyclically pure in an $R$-algebra $S$, then $R$ is pure in $S$. As it turns out, the "approximately Gorenstein" condition we discuss below is not only sufficient for this purpose but also necessary, and guarantees the desired conclusion even when $S$ is merely a module, without an algebra structure.

But the definition of "approximately Gorenstein" leaves much to be desired: the hard job is to give a more down-to-earth characterization of this notion. It turns out to be a rather weak (albeit strange) unmixedness condition.

A good deal of our effort will be devoted to proving the existence of $m$ primary irreducible ideals contained in arbitrarily high powers of the maximal ideals of certain Noetherian local rings $(R, m)$ : the existence of such irreducible ideals is a subtle question and is the key to all our difficulties. It turns out to be necessary to consider an analogous question for modules as well (see Theorem 1.12). Matlis duality [17] plays a crucial role at a certain point, and so does the notion of "canonical module". The use of canonical modules has been quite minimized in this write-up: they played a much larger role in the original thinking. It will still be evident (see Example (5.4)) that the existence of canonical modules and the existence of small irreducibles are closely related questions, and we shall later give an example, based on the Ferrand-Raynaud example [5], in which cyclic purity fails to imply purity for a one-dimensional local Noetherian domain $R$.

We note that some work on the comparison of cyclic purity and purity has been done in [2] and [9] (and cyclic purity is studied in [8]). Where we say that $R$ is "cyclically pure" or "ideally closed" in $S$, in [9] $R \rightarrow S$ is said to satisfy "condition C". Where we refer to the "purity" of $R \rightarrow S$, [9] refers to "condition $\xi$ " (essentially, P. M. Cohn's linear equational criterion for purity: see [3] or [20, p. 65]).

1. The main notions and the main results. We shall write $\operatorname{dim} R$ for the Krull dimension of $R$.

Let $(R, m)$ be a Noetherian local ring with $\operatorname{dim} R=n$. We recall ([1], [16]) that $R$ is Gorenstein if, equivalently, either:

(i) $\mathrm{id}_{R} R$ is finite (id denotes injective dimension); in this case, $\mathrm{id}_{R} R=n$, or

(ii) some (respectively, every) system of parameters $x_{1}, \ldots, x_{n}$ is an $R$ sequence such that $\left(x_{1}, \ldots, x_{n}\right) R$ is irreducible, or

(iii) $R$ is Cohen-Macaulay and for some (respectively, every) system of parameters $x_{1}, \ldots, x_{n}$ of $R, S=R /\left(x_{1}, \ldots, x_{n}\right) R$ is an injective $S$-module.

An arbitrary Noetherian ring $R$ is Gorenstein if all its local rings are.

We are now ready for our most important definition:

(1.1) Definition-Proposition. A local Noetherian ring $(R, m)$ is approximately Gorenstein if it satisfies either of the following equivalent conditions: 
(i) For every integer $N>0$ there is an ideal $I \subset m^{N}$ such that $R / I$ is Gorenstein.

(ii) For every integer $N>0$ there is an m-primary irreducible ideal $I \subset m^{N}$.

[For a proof, see (2.1) and (2.2) of \$2.]

(1.2) Proposition. A local Noetherian ring $(R, m)$ is approximately Gorenstein if and only if its m-adic completion $(\hat{R}, \hat{m})$ is approximately Gorenstein.

[For a proof, see (2.2) of $\S 2$.]

(1.3) Definition. A Noetherian ring $R$ is approximately Gorenstein if for every maximal ideal $m$ of $R, R_{m}$ is approximately Gorenstein.

The "propositions" above are rather trivial. The next proposition is more interesting: it displays the connection between approximately Gorenstein rings and the original problem of getting cyclic purity to imply purity.

(1.4) Proposition. Let $R$ be a Noetherian ring. The following three conditions on $R$ are equivalent:

(i) $R$ is approximately Gorenstein.

(ii) $R$ is pure in every extension algebra $S \supset R$ such that $R$ is cyclically pure (i.e., ideally closed) in $S$.

(iii) $R$ is pure in every extension module $M \supset R$ such that $R$ is cyclically pure in $M$.

[For a proof, see (2.6) of $\S 2$.]

Examples of ring extensions $R \subset S$ such that $R$ is Noetherian (even Artinian), $R$ is cyclically pure in $S$, yet $R$ is not pure in $S$ abound. We shall give examples below: see $\$ 3$ and Example (5.4). The reader may wish to consult [4] and [2] where other examples in which cyclic purity holds but purity fails are given. In the example of [2], $R$ is Artinian and $S$ is a finite $R$ module.

On the other hand, when $R$ is excellent Noetherian a rather weak unmixedness condition is sufficient for cyclic purity to imply purity if $\operatorname{dim} R \geqslant 2$. By virtue of (1.4) and (1.2) the problem is basically to characterize the approximately Gorenstein rings in the complete local case. If $\operatorname{dim} R=0$ we have a trivial result:

(1.5) Proposition. If $R$ is an Artin local ring, then $R$ is approximately Gorenstein if and only if $R$ is Gorenstein.

[See (2.26).]

If $\operatorname{dim} R \geqslant 1$ we have our first main theorem:

(1.6) ThEOREM. Let $(R, m)$ be a complete (or even an excellent) local ring with 
$\operatorname{dim} R \geqslant 1$. Then $R$ is approximately Gorenstein if and only if the following two conditions hold:

(a) $m \notin \operatorname{Ass}(R)$, i.e., $\operatorname{depth} R \geqslant 1$.

(b) If $P \in \operatorname{Ass}(R)$ and $\operatorname{dim} R / P=1$, then $(R / P) \oplus(R / P)$ is not embeddable in $R$.

[See (5.2) for a proof.]

For basic properties of excellent rings we refer the reader to [18]. We merely mention here that the class of excellent Noetherian rings contains all fields, the integers, all discrete valuation rings of characteristic 0 , and is closed under passage to a residue class ring, passage to a ring of quotients with respect to a multiplicative system, and passage to a finitely generated extension algebra. For other properties we shall refer to [18] as needed.

For the purpose of applying (1.6) the following corollary is really our main result (note: by " $R$ is locally excellent" we mean the local rings of $R$ are excellent):

(1.7) THEOREM. Let $R$ be any locally excellent Noetherian ring and suppose that at least one of the following three conditions holds:

(1) $R$ is reduced (every nilpotent is 0 ), or

(2) $R$ is generically Gorenstein (i.e., the total quotient ring of $R$ is a (necessarily 0 -dimensional) Gorenstein ring), or

(3) for every prime $P \in \operatorname{Ass}(R)$ and maximal ideal $m \supset P, \operatorname{dim}(R / P)_{m} \geqslant 2$.

Let $S$ be any extension algebra of $R$ such that $R$ is cyclically pure ( $\equiv$ ideally closed) in $S$. Then $R$ is pure in $S$. Hence, if $S$ is also a finite $R$-module, then if $R$ is cyclically pure in $S, R$ is a direct summand of $S$ as an $R$-module.

[See (5.3) for a proof.]

This result is not best possible, but conditions (1) or (2) or (3) are the most easily verified in practice. Note that $(1) \Rightarrow(2)$, since if $R$ is reduced its total quotient ring is a finite product of fields. In general, if $R$ is generically Gorenstein its total quotient ring will be a semilocal zero-dimensional Gorenstein ring, and, hence, a finite product of zero-dimensional Gorenstein local rings.

The key point will be to prove that a "good" $R$ has sufficiently many irreducibles. We proceed by working with modules, which is both natural and necessary. Before stating our main result for modules, we give two convenient definitions:

(1.8) Definition. We shall say that a local ring $A$ is an $E$-ring if every domain $B$ which is a homomorphic image of $A$ satisfies the following condition: if $\operatorname{dim} B=1$, then the generic fiber $(B-\{0\})^{-1} \hat{B}$ of $B \rightarrow \hat{B}$ is Gorenstein, while if $\operatorname{dim} B \geqslant 2$ then $\operatorname{Ass}(\hat{B})$ contains no primes of coheight one. 
We say that an arbitrary Noetherian ring $R$ is an $E$-ring if its localization at each maximal ideal is an $E$-ring.

The key point that we need about excellent rings is simply:

(1.9) Proposition. A (locally) excellent Noetherian ring is an E-ring. Hence, a complete local ring is an E-ring.

ProOF. See [18, §34].

(1.10) Definition. Let $(R, m)$ be a local ring and let $M$ be an $R$-module of finite type. We shall say that $M$ has small cofinite irreducibles (S.C.I.) if for every integer $N>0$ there is an irreducible submodule $E$ of $M$ such that $E \subset m^{N} M$ and $M / E$ has finite length.

By $\operatorname{dim} M$, where $M$ is an $R$-module, we mean $\operatorname{dim}\left(R / \operatorname{Ann}_{R} M\right)$. One has, trivially:

(1.11) Proposition. Let $(R, m)$ be local Noetherian and $M$ an $R$-module of finite type. Then:

(a) $M$ has S.C.I. if and only if $\hat{M}$ has S.C.I. over $\hat{R}$.

(b) If $\operatorname{dim} M=0$, then $M$ has S.C.I. if and only if 0 is irreducible in $M$.

(c) $M=R$ has S.C.I. if and only if $R$ is approximately Gorenstein.

Our main result on modules having S.C.I. is the following:

(1.12) TheOREM. Let $(R, m)$ be a local Noetherian ring and let $M$ be an $R$ module of finite type with $\operatorname{dim} M>0$. Consider the following condition on $M$ :

(*) $m \notin \operatorname{Ass}(M)$ (i.e., depth $M \geqslant 1$ ) and if $P \in \operatorname{Ass}(M)$ is such that $\operatorname{dim}(R / P)=1$, then $(R / P) \oplus(R / P)$ cannot be embedded in $M$.

If $M$ has small cofinite irreducibles, then $M$ satisfies (*), while if $R$ is an E-ring then $M$ has S.C.I. if and only if $M$ satisfies (*).

[For a proof, see (4.4), (4.11), and (4.17).]

This result is really the strongest of our theorems: it gives (1.6) and (1.7) at once. Moreover, the following proposition shows that (1.12) is best possible in a certain sense and justifies the notion of an $E$-ring:

(1.13) Proposition. Let $(R, m)$ be a local ring and suppose that every $R$-module $M$ of finite type such that $\operatorname{dim} M>0$ which satisfies (*) has small cofinite irreducibles. Then $R$ is an E-ring. Thus, $R$ is an E-ring if and only if (*) is equivalent to having S.C.I. for positive dimensional $M$.

[For a proof, see (4.12).]

We also note the following easy consequence of (1.6) and (1.7).

(1.14) Proposition. Let $R$ be an arbitrary Noetherian ring and let $x, y$ be analytic indeterminates over $R$. Then $R[x, y]$ and $R[[x, y]]$ are approximately Gorenstein. 
Moreover, given a ring extension $R \rightarrow S$, where $R$ is Noetherian, $R \rightarrow S$ is pure if and only if $R[x, y] \rightarrow S[x, y]$ is cyclically pure if and only if $R[[x, y]] \rightarrow S[[x, y]]$ is cyclically pure.

[For a proof, see (5.6).]

On the other hand, we note the following result from $\S 6$.

(1.15) Proposition. There exists a pure ring extension $R \rightarrow S$ such that $R[[t]] \rightarrow S[[t]]$ is not even cyclically pure: in fact principal ideals are not concerned.

[See (6.1).]

We conclude this summary of our main results with the remark that in $\$ 3$ it is shown that if $R$ is a 0 -dimensional local Noetherian ring which is not Gorenstein, it has a sort of "universal" or "generic" finitely generated extension algebra $T_{R}$ such that $R \rightarrow T_{R}$ is not pure. Moreover, $R$ is cyclically pure but not pure in $T_{R}$.

2. Approximately Gorenstein rings, cyclic purity, and purity. The following proposition implies the results in (1.1) and (1.2) of $\S 1$.

(2.1) Proposition. Let $(R, m)$ be a local Noetherian ring, $N>0$ an integer, and suppose that $I \subset m^{N}$ and $R / I$ is Gorenstein. Then there exists an ideal $J$ with $I \subset J \subset m^{N}$ such that $J$ is $m$-primary and, equivalently:

(1) $J$ is irreducible.

(2) $R / J$ is Gorenstein.

(3) $\operatorname{Soc}(R / J) \cong R / m$ (where "Soc" denotes "Socle").

Proof. Let $k=\operatorname{dim}(R / I)$ and let $x_{1}, \ldots, x_{k} \in m$ be such that $\bar{x}_{1}, \ldots, \bar{x}_{k}$ is a system of parameters for $R / I$, where - denotes reduction modulo $I$. Then $\bar{x}_{1}^{N}, \ldots, \bar{x}_{k}^{N}$ is also a system of parameters for $R / I$, and $(R / I) /\left(\bar{x}_{1}^{N}, \ldots, \bar{x}_{k}^{N}\right)$ is a zero-dimensional Gorenstein ring. Hence, we may let $J=I+\left(x_{1}^{N}, \ldots\right.$, $\left.x_{k}^{N}\right) R$. Q.E.D.

(2.2) COROLlaRy. (a) The conditions given in (1.1) (defining "approximately Gorenstein") are equivalent.

(b) Gorenstein rings are approximately Gorenstein. A zero-dimensional ring is approximately Gorenstein if and only if it is Gorenstein.

(c) A local Noetherian ring $(R, m)$ is approximately Gorenstein if and only if its $m$-adic completion $(\hat{R}, \hat{m})$ is approximately Gorenstein.

Proof. In (1.1) the implication (ii) $\Rightarrow$ (i) is clear, while (i) $\Rightarrow$ (ii) is immediate from (2.1) above. The first statement in (b) is clear from characterization (i) of approximately Gorenstein rings. For the second statement of (b) we need only consider the case of a zero-dimensional Noetherian local ring 
$(R, m)$. Choose $N$ so that $m^{N}=0$. If $R$ is approximately Gorenstein, we can choose $I \subset m^{N}$ so that $R / I=R /(0) \cong R$ is Gorenstein.

Finally, statement (c) is clear from characterization (ii) of approximately Gorenstein rings. Q.E.D.

We next want to give a result which contains Proposition (1.4). It will be convenient to make some definitions:

(2.3) Definition. If $R$ is a Noetherian ring, $g(R)=\{I \subset R: I$ is an irreducible ideal and $\operatorname{Rad}(I)$ is a maximal ideal of $R$ \}.

(2.4) Definition. If $R$ is a Noetherian ring, $\delta(R)$ (or simply $\delta$ ) denotes the $R$-algebra $\Pi_{I \in \mathcal{G}(R)} R / I$.

We also note the following trivial fact:

(2.5) LeMMA. Let $R$ be a Noetherian ring. If $r \in R, r \neq 0$, and I is maximal with respect to not containing $r$, then $I \in g(R)$. Hence, for any Noetherian ring $R, \cap_{I \in G(R)} I=(0)$, i.e., $R \rightarrow \Im(R)$ is injective.

The proof is left to the reader. We are now ready for the main result of this section:

(2.6) TheOREM. Let $R$ be Noetherian ring. Then $R \rightarrow \delta(R)$ is ideally closed, and the following four conditions are equivalent:

(i) $R$ is approximately Gorenstein.

(ii) $R \rightarrow \mathfrak{S}(R)$ is pure.

(iii) For every extension algebra $S$ of $R$, if $R$ is ideally closed ( $\equiv$ cyclically pure) in $S$, then $R$ is pure in $S$.

(iv) For every extension module $M$ of $R$, if $R \rightarrow M$ is cyclically pure, then $R$ is pure in $M$.

Proof. We shall show that (iv) $\Rightarrow$ (iii) $\Rightarrow$ (ii) $\Rightarrow$ (i) $\Rightarrow$ (iv). (iv) $\Rightarrow$ (iii) is trivial, while (iii) $\Rightarrow$ (ii) is immediate if we know that $R \rightarrow \delta$ is ideally closed. To see this suppose, to the contrary, that $I \subset R, r \in R-I$, and $r \in I S$. Choose $J \supset I$ maximal with respect to not containing $r$. By $(2.5), J \in g(R)$. If we compose $R \rightarrow \delta \rightarrow^{p} R / J$, where $p$ is the product projection for the $J$ coordinate, then since $r \in I S \subset J \delta$, the image of $r$ is 0 in $R / J$, contradicting $r \notin J$. Thus, $R \rightarrow \delta(R)$ is ideally closed, and (iii) $\Rightarrow$ (ii).

To see that (ii) $\Rightarrow$ (i), assume that $R \rightarrow \delta(R)$ is pure and let $m$ be a given maximal ideal of $R$. Let $N$ be a given integer $>0$. Let $K=R / m$ and let $E$ denote the injective hull of $K$ as a module over $B=R / m^{n}$. Then, using the basic facts about Matlis duality [17], we have that $E$ has finite length as a $B$ module and, hence, $E$ is finitely generated ( $\Rightarrow$ finitely presented) as an $R$ module. Moreover, $\operatorname{Hom}_{R}(E, E)=\operatorname{Hom}_{B}(E, E) \cong B$ (the last isomorphism is the crucial consequence of Matlis duality which we need here), where under $B \cong \operatorname{Hom}_{B}(E, E)$ the element $b$ maps to multiplication by $b$. 
Since $R \rightarrow \delta$ is pure, the map $E \rightarrow E \otimes_{R} \delta=E \otimes_{R} \Pi_{I}(R / I)$ is injective. Now, quite generally, if $R$ is any commutative ring, $E$ is a finitely presented $R$ module, and $\left\{Q_{\lambda}\right\}_{\lambda \in \Lambda}$ is an arbitrary family of $R$-modules, the natural map

$$
E \otimes_{R}\left(\prod_{\lambda} Q_{\lambda}\right) \rightarrow \prod_{\lambda}\left(E \otimes_{R} Q_{\lambda}\right)
$$

is an isomorphism. [The map takes $e \otimes f$, where $f: \Lambda \rightarrow \cup_{\lambda} Q_{\lambda}$ is such that $f(\lambda) \in Q_{\lambda}$, to $g$, where $g(\lambda)=e \otimes f(\lambda)$.] This gives a natural transformation from the right exact functor $F=\cdot \otimes_{R}\left(\Pi_{\lambda} Q_{\lambda}\right)$ to the right exact functor $G=\Pi_{\lambda}\left(\cdot \otimes_{R} Q_{\lambda}\right)$. Hence, to check that $F(E) \rightarrow G(E)$ is an isomorphism for finitely presented $E$, it suffices to check the case $E=R^{m}$. But then the natural transformation is given by $F(E)=R^{m} \otimes_{R}\left(\Pi_{\lambda} Q_{\lambda}\right) \cong\left(\Pi_{\lambda} Q_{\lambda}\right)^{m}$ $\cong \Pi_{\lambda}\left(Q_{\lambda}^{m}\right)=\Pi_{\lambda}\left(R^{m} \otimes_{R} Q_{\lambda}\right)=G(E)$. Hence, $E \otimes_{R} \delta \cong \Pi_{I}\left(E \otimes_{R} R / I\right)$ $\cong \Pi_{I}(E / I E)$, where $I \in G(R)$, and we have that $E \rightarrow \prod_{I}(E / I E)$ is injective. Let $u$ generate the copy of $K$ in $E$, i.e., $R u \cong K \hookrightarrow E$. Then we can choose $I \in g(R)$ such that $K \cong R u \nsubseteq I E$. But $R u$ is contained in every nonzero submodule of $E$. Hence, we can choose $I \in g(R)$ such that $I E=0$. But then $I \subset m^{N}$ as required, for if $r \in I-m^{N}$ then $\bar{r} \neq 0$ in $B=R / m^{N}\left({ }^{-}\right.$denotes reduction module $m^{N}$ ) and, hence, by Matlis duality, multiplication by $r$ or $\bar{r}$ gives a nonzero endomorphism of $E\left(B \cong \operatorname{Hom}_{B}(E, E)\right)$. This says that $r E \neq 0$ and so $I E \neq 0$, a contradiction. This completes the proof that (ii) $\Rightarrow$ (i).

It remains to show that (i) $\Rightarrow$ (iv). Suppose that $R$ is approximately Gorenstein and that $0 \rightarrow R \rightarrow M$ is cyclically pure. We must show that for every $R$-module $E, E=R \otimes E \rightarrow M \otimes E$ is injective. Since $E$ is a direct limit of finitely generated modules, we may assume that $E$ is finitely generated. Suppose $e \neq 0$ in $E$ but $1 \otimes e$ is 0 in $M \otimes E$. Let $m$ be a maximal ideal of $R$ which contains $\mathrm{Ann}_{R} R e .0 \rightarrow R e \rightarrow E$ is injective, and hence $0 \rightarrow(R e)_{m}$ $\rightarrow E_{m}$ is injective, and $(R e)_{m} \neq 0$. Choose $N$ such that $e / 1$ is not in $m^{N} E_{m}$ : this is possible since $e / 1 \neq 0$ and $\cap_{t} m^{t} E_{m}=0$. Then the image of $e$ in $E_{m} / m^{N} E_{m} \cong E / m^{N} E$ is not 0 . We have a commutative diagram:

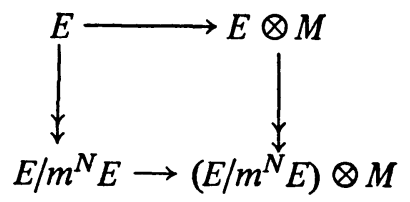

Since $e$ maps to 0 in $E \otimes M$, its image in $E / m^{N} E$ (is nonzero) but maps to 0 in $\left(E / m^{N} E\right) \otimes M$. Thus, purity fails for a module $\bar{E}$ which is killed by $m^{N}$ for a certain $m, N$. But this is impossible: choose $I \subset m^{N}$ so that $I$ is $m$-primary and irreducible ( $R$ is approximately Gorenstein, which permits us to do this). 
Then $m^{N} \bar{E}=0 \Rightarrow I \bar{E}=0 \Rightarrow \bar{E} \cong R / I \otimes_{R} \bar{E}$; moreover, $B=R / I$ is zerodimensional Gorenstein and, hence, $B$-injective. Since $0 \rightarrow R \rightarrow M$ is cyclically pure, $0 \rightarrow B \rightarrow B \otimes M$ is exact, and then since $B$ is $B$-injective, $B$ is a direct summand of $B \otimes M$ as $B$-modules, whence $0 \rightarrow E \otimes_{B} B \rightarrow E \otimes_{B}\left(B \otimes_{R} M\right)$ is injective, and since $I E=0$, this sequence may be identified with $0 \rightarrow E$ $\rightarrow E \otimes_{R} M$. Q.E.D. for (i) $\Rightarrow$ (iv) and Theorem 2.6.

(2.7) ReMARK. The fact that $R$ is approximately Gorenstein does not imply that $R_{P}$ is approximately Gorenstein for every $P$. It is easy to see this if we allow ourselves the use of the characterization of Theorem 1.6. Let $R$ $=K\left[\left[x_{1}, x_{2}, y_{1}, y_{2}\right]\right] /\left(y_{1}, y_{2}\right)^{2}$, where $K$ is any field. Then $R$ is approximately Gorenstein, because the unique prime $P$ in $\operatorname{Ass}(R)$ (which is generated by the images of $y_{1}, y_{2}$ ) is of coheight 2. But $R_{P}$ is zero-dimensional and not Gorenstein, and hence not approximately Gorenstein.

(2.8) Remark. Suppose we had allowed irreducibles whose primes are nonmaximal in the construction of $\delta$ and had then tried to prove, instead of (ii) $\Rightarrow$ (i) of (2.6), that given (ii), $R_{P}$ is approximately Gorenstein for every prime $P$ of $R$. Then we immediately run into the stumbling block that the injective hull of $R_{P} / P R_{P}$ as an $\left(R_{P} / P^{n} R_{P}\right)$-module is not of finite type over $R$, and we cannot distribute $\otimes$ over the infinite product of modules as in the proof of (2.6).

(2.9) Remark. The implication (i) $\Rightarrow$ (iv) is essentially given, at least implicitly, in the local case, in [12]. The ideas of [12] motivated our work here, while [12] in turn was motivated by the relationship between results on contractedness and the existence of big Cohen-Macaulay modules: this relationship is discussed in [13].

\section{Generic nonpure extension algebras of Artin local rings.}

(3.1) Proposition. Let $(R, m)$ be an Artin local ring, let $K=R / m$, and let $A$ be an $s$ by $t$ matrix with entries in $m$ such that $\operatorname{Coker}\left(A: R^{s} \rightarrow R^{t}\right)$ is an injective hull $E$ for $K$ (we may obtain such a matrix from a minimal free $R$-resolution of an injective hull of $K)$. Let $X=\left[X_{1} \cdots X_{s}\right]$ be an $s \times 1$ matrix of indeterminates, let $S=R\left[X_{1}, \ldots, X_{s}\right]$, let $p \in R^{t}$ map to an element of $E$ which generates the copy of $K$ in $E$, and let $J$ be the ideal of $S$ generated by the entries of $p-X A$. Let $T_{R}=S / J$. Then:

(a) $T_{R}$ is a finitely generated $R$-algebra. Its $R$-algebra structure is independent of the choice of $A$ or $P$.

(b) $R \rightarrow T_{R}$ is not pure, and is "generic" with respect to this property in the sense that $h: R \rightarrow U$ is not pure if and only if there is a homomorphism $T_{R} \rightarrow U$ such that $h=\left(T_{R} \rightarrow U\right) \circ\left(R \rightarrow T_{R}\right)$.

(c) If $R$ is not Gorenstein, $R \rightarrow T_{R}$ is injective and cyclically pure ( $R$ is ideally 
closed in $T_{R}$ ). If $R$ is Gorenstein, $s=0, S=R$, and $T_{R} \cong R / \operatorname{Ann}_{R} m ; R \rightarrow T_{R}$ is not injective.

Proof. (a) Consider one choice of $A, p$. Any other choice of $A$ has the form $B A C$, where $B$ is $s$ by $s$ invertible, $C$ is $t$ by $t$ invertible, and then we may initially choose the new $p$ to be $p C$. Let $X^{\prime}=X B$. Then the entries $X_{1}^{\prime}, \ldots, X_{s}^{\prime}$ of $X^{\prime}$ are indeterminates over $R$ and $S=R\left[X_{1}^{\prime}, \ldots, X_{s}^{\prime}\right]$. The new algebra $T_{R}^{\prime}$ obtained is $S / J^{\prime}$, where $J^{\prime}$ is generated by the entries of $p C-X(B A C)$ $=p C-(X B)(A C)=(p-(X B) A) C$, and since $C$ is invertible $J^{\prime}$ is generated by the entries of $p-X^{\prime} A$ and

$$
T_{R}^{\prime}=R\left[X_{1}^{\prime}, \ldots, X_{s}^{\prime} V\left(\text { the entries of } p-X^{\prime} A\right) \cong T_{R} .\right.
$$

Now suppose that $A$ is fixed but we vary $p$. Any choice of $p$ has the form $p^{\prime}=a p+D A$ where $a$ is an invertible element of $R$ and $D$ is a 1 by $s$ matrix of elements of $R$, say $D=\left[D_{1} \cdots D_{s}\right]$. In this case $T_{R}^{\prime}=S / J^{\prime}$, where $J^{\prime}$ is generated by the entries of $a p+D A-X A=a\left(p-a^{-1}(X-D) A\right)$, and hence $J^{\prime}$ is generated by the entries of $p-X^{\prime} A$, where, now, $X^{\prime}=$ $a^{-1}(X-D)$. Since the $X_{i}^{\prime}=a^{-1}\left(X_{i}-D_{i}\right)$ are algebraically independent over $R$ and generate $S$, we again have $T_{R}^{\prime} \cong T_{R}$.

Of course, it is obvious that $T_{R}$ is finitely generated over $R$.

(b) Let $\phi: K \rightarrow E$ be the map which takes $1+m$ to the image $\bar{p}$ of $p$. By [14, Proposition 6.11], $h: R \rightarrow U$ is pure if and only if the induced map $K \otimes U$ $\rightarrow E \otimes U$ is injective, i.e., if and only if $\bar{p} \otimes 1$ is nonzero in $E \otimes U$. Since $E=\operatorname{Coker} A$ (over $R$ ), $E \otimes U \cong \operatorname{Coker} h(A)$ (over $U$ ), and $\bar{p} \otimes 1$ is represented by the image of $h(p)$ in Coker $h(A)$. Then, $h$ is not pure $\Leftrightarrow h(p)$ represents 0 in Coker $h(A) \Leftrightarrow h(p)$ is in the row space of $h(A) \Leftrightarrow$ there exists an $s \times 1$ matrix $\bar{X}$ over $U$ such that $h(p)-\bar{X} h(A)=0 \Leftrightarrow$ there is a homomorphism $S=R\left[X_{1}, \ldots, X_{s}\right] \rightarrow U$ extending $h: R \rightarrow U$ (corresponding to $X \mapsto \bar{X}$ ) such that $p-X A$ maps to $0 \Leftrightarrow h: R \rightarrow U$ factors through $S / J=T_{R}$, as required.

(c) If $R$ is not Gorenstein, then by (2.2b) $R$ is not approximately Gorenstein, and by Theorem 2.6, $R \rightarrow \delta(R)$ is injective, ideally closed, but not pure. Hence, this map has a factorization $R \rightarrow T_{R} \rightarrow \delta(R)$, by part (b) here. This implies $R \rightarrow T_{R}$ is injective and ideally closed.

If $R$ is Gorenstein, $E=R$ and the minimal resolution is $0 \rightarrow R \rightarrow R \rightarrow 0$, i.e., $s=0, t=1$. We interpret this to mean that $S=R$ (there are 0 indeterminates). $p$ is then, simply, an element which generates the unique copy of $K \cong \mathrm{Ann}_{R} m$ in $R$, and we regard the product of the 1 by 0 matrix $X$ and the 0 by 1 matrix $A$ to be the $1 \times 1$ matrix [0], so that $J$ is generated by $p-0=p$. Q.E.D.

(3.2) REMARK. If $(R, m)$ is local one cannot get a single algebra to play the role of $T_{R}$, because the injective hull $E$ of $R / m$ is countably generated rather than finitely generated. 
(3.3) Example. Let $R=K[X, Y] /(X, Y)^{2}=K[x, y](=K+K x+K y)$, where $K$ is any field. In this case, we may take

$$
A=\left[\begin{array}{cc}
y & 0 \\
0 & x \\
x & -y
\end{array}\right]
$$

and $p=\left[\begin{array}{ll}x & 0\end{array}\right]\left(\equiv\left[\begin{array}{ll}0 & y\end{array}\right]\right)$. Then

$$
p-X A=\left[x-X_{1} y-X_{3} x,-X_{2} x+X_{3} y\right]
$$

and

$$
T_{R}=R\left[X_{1}, X_{2}, X_{3}\right] /\left(x-X_{1} y-X_{3} x,-X_{2} x+X_{3} y\right) .
$$

Then $R$ is not pure in $T_{R}$, and since $R$ is not Gorenstein $R$ is ideally closed in $T_{R}$, by Proposition 3.1. But it is easy to see this directly: except for (0) and $m$ (which are easily checked separately), every proper ideal of $R$ is irreducible and is, in fact, the kernel of a surjective homomorphism $h: R \rightarrow K[t]$ $\cong K[T] /\left(T^{2}\right) . h$ is uniquely determined by elements $a, b \in K$, where $(a, b)$ $\neq(0,0)$, and $h(x)=a t, h(y)=b t$. The assertion that $\left((\operatorname{Ker} h) T_{R}\right) \cap R$ $=\operatorname{Ker} h$ is equivalent to the injectivity of the map

$$
K[t] \rightarrow K[t]\left[X_{1}, X_{2}, X_{3}\right] /\left(a t-X_{1} b t-X_{3} a t,-X_{2} a t+X_{3} b t\right)
$$

induced by substituting $x=a t, y=b t$. If $a=0$ this injectivity is clear: we may further compose with a map that kills $\bar{X}_{1}, \bar{X}_{2}, \bar{X}_{3}$. If $a \neq 0$ we may compose with a map which sends $\bar{X}_{1}$ to $0, \bar{X}_{3}$ to 1 , and $\bar{X}_{2}$ to $b a^{-1}$. In either case the target ring may be retracted to $K[t]$.

4. The existence of small cofinite irreducibles. In this section, which is the heart of the paper, we prove the key theorem, (1.12). We proceed by giving a sequence of lemmas and propositions which eventually yields the desired result.

Throughout the rest of this section, unless otherwise specified, $(R, m)$ denotes a Noetherian local ring and $K=R / m$. ^ denotes $m$-adic completion. $M$ and $E$ always denote $R$-modules of finite type. (Some of these hypotheses may be reiterated, for emphasis, in the statements of theorems.)

(4.1) Proposition. (a) If $\operatorname{dim} M=0, M$ has small cofinite irreducibles if and only if 0 is irreducible in $M$, i.e., $M=0$ or $M \neq 0$ and $\operatorname{Hom}_{R}(K, M) \cong K$.

(b) $M$ has S.C.I. over $R$ if and only if $\hat{M}$ has S.C.I. over $\hat{R}$.

(c) If $I \subset \mathrm{Ann}_{R} M$, then $M$ has S.C.I. over $R / I$ if and only if $M$ has S.C.I. over $R$.

The proof is utterly straightforward and is left to the reader. 
(4.2) Proposition. $M$ has small cofinite irreducibles if and only if for every integer $N>0$ there is a submodule $M_{N} \subset m^{N} M$ such that $M / M_{N}$ has S.C.I.

Proof. "Only if" is clear, from the definition of having S.C.I. and (4.1a). To prove "if", let $N>0$ be given and choose $M_{N} \subset m^{N} M$ such that $M / M_{N}$ has S.C.I. Choose $E \subset m^{N}\left(M / M_{N}\right)$ such that $\left(M / M_{N}\right) / E$ has finite length and $E$ is irreducible in $M / M_{N}$ (i.e., 0 is irreducible in $\left(M / M_{N}\right) / E$ ). Let $M_{N}^{\prime}$ be the inverse image of $E$ in $M$, so that $M / M_{N}^{\prime} \cong\left(M / M_{N}\right) / E$. Then $M_{N}^{\prime} \subset m^{N} M$ $+M_{N} \subset m^{N} M$ and $M_{N}^{\prime}$ is cofinite (i.e., $M / M_{N}$ has finite length), while $M_{N}^{\prime}$ is irreducible in $M$ because 0 is irreducible in $M / M_{N}^{\prime} \cong\left(M / M_{N}\right) / E$. Q.E.D.

(4.3) Proposition. Suppose that $M$ has S.C.I. Then every submodule $E$ of $M$ has S.C.I.

Proof. By the Artin-Rees lemma there is a positive integer $c$ such that for every integer $t>0,\left(m^{t+c} M\right) \cap E \subset m^{t} E$. Let $N>0$ be given and choose $M^{\prime} \subset m^{N+c} M$ such that $M / M^{\prime}$ has finite length and $M^{\prime}$ is irreducible in $M$. Let $E_{N}=M^{\prime} \cap E$. Then $E_{N} \subset\left(m^{N+c} M\right) \cap E \subset m^{N} E$. Moreover, we have an injection $E / E_{N} \hookrightarrow M / M^{\prime}$. Since $M / M^{\prime}$ has finite length and 0 is irreducible in it, every submodule of $M / M^{\prime}$ has the same property. Thus, $E_{N}$ is cofinite and irreducible in $E$. Q.E.D.

We are now ready to begin the proof of the necessity of condition (*) for a module to have S.C.I. (see (1.12)). First:

(4.4) Proposition. If $M$ has S.C.I. and $\operatorname{dim} M \geqslant 1$ then $\operatorname{depth} M \geqslant 1$, i.e., $m \notin \operatorname{Ass}(M)$.

Proof. If depth $M=0$, choose $x \neq 0$ in $M$ such that $m x=0$. Since $x \neq 0$ we can choose $N>0$ such that $x \notin m^{N} M$. Choose $M^{\prime} \subset m^{N+1} M$ such that $M^{\prime}$ is cofinite and irreducible in $M$. Since $x \notin M^{\prime}$ and $m x=0$, $x+M^{\prime}$ generates $\operatorname{Soc}\left(M / M^{\prime}\right)=\operatorname{Ann}_{M / M^{\prime}} m\left(\cong K\right.$, since $M^{\prime}$ is irreducible). Since $\operatorname{dim} M \geqslant 1, m^{N} M \neq m^{N+1} M$. Let $y \in m^{N} M-m^{N+1} M$. Since $y$ $\notin M^{\prime}, y+M^{\prime}$ has a multiple equal to $x+M^{\prime}$, i.e., there exists $r \in R$ such that $x-r y \in M^{\prime} \subset m^{N+1} M$. Since $y \in m^{N} M, x \in m^{N} M+m^{N+1} M$ $=m^{N} M$, a contradiction. Q.E.D.

Before continuing the proof of the necessity of $(*)$, we recall some needed facts about canonical modules in the local case.

(4.5) Discussion. Let $(R, m)$ be a Cohen-Macaulay local ring, with $\operatorname{dim} R$ $=n$. A canonical module $E$ for $R$ is an $R$-module of finite type satisfying the following condition: if $x_{1}, \ldots, x_{n}$ is a system of parameters for $R$ (equivalently, a maximal $R$-sequence) then $x_{1}, \ldots, x_{n}$ is an $E$-sequence and $E /\left(x_{1}, \ldots, x_{n}\right) E$ is an injective hull for $K$ regarded as an $\left(R /\left(x_{1}, \ldots, x_{n}\right) R\right)$ module. $E$ is unique up to nonunique isomorphism, if it exists. However, the natural map $R \rightarrow \operatorname{Hom}_{R}(E, E)$ is an isomorphism, so that the isomorphism 
between two canonical modules is unique up to multiplication by a unit of $R$.

A Cohen-Macaulay ring $R$ possesses a canonical module if and only if $R$ is a homomorphic image of a Gorenstein local ring [19], [7], [6]: a complete local Cohen-Macaulay ring always has one. $R$ is Gorenstein if and only if it is a canonical module for itself. See [22] and [23] for more information. (Note: A "canonical module" is a "rank one Gorenstein module" in the terminology of some authors.)

We note that if $R$ is of finite type over (perhaps a homomorphic image of) a Gorenstein local ring $S$ of dimension $q \geqslant n$, then $\operatorname{Ext}_{S}^{q-n}(R, S)$ is a canonical module for $R$.

If $E$ is a canonical module for $R$ and $x \in R$ is not a zerodivisor, then $x$ is not a zerodivisor on $E$ and $E / x E$ is a canonical module for $R / x R$.

If $P$ is a prime ideal of $R$, then $E_{P}$ is a canonical module for $R_{P}$.

We refer the reader to [7], [10], [11], [22], and [23] for further information and details.

We also need the following fact from [11]:

(4.6) FACT. Let $(R, m)$ be a Cohen-Macaulay ring which possesses a canonical module $E$. Let $x_{1}, \ldots, x_{n}$ be a system of parameters for $R$. Then

$$
W=\operatorname{inj} \lim _{t}\left(\cdots \rightarrow E /\left(x_{1}^{t}, \ldots, x_{n}^{t}\right) E \stackrel{x_{1} \cdots x_{n}}{\longrightarrow} E /\left(x_{1}^{t+1}, \ldots, x_{n}^{t+1}\right) E \rightarrow \cdots\right)
$$

is an injective hull for $K$ over $R$.

Proof. $E /\left(x_{1}^{t}, \ldots, x_{n}^{t}\right) E$ is an injective hull of $K$ over $R /\left(x_{1}^{t}, \ldots, x_{n}^{t}\right) R$, and, since $x_{1}, \ldots, x_{n}$ is an $E$-sequence, the maps are injective. It follows that the modules in the sequence fit together to give the injective hull of $K$ over $R$. (Alternatively, by [11, Theorem 2.3], $W=H_{m}^{n}(E)=H_{m}^{n}(\hat{E})$, is an injective hull of $K$ over $R$ or $\hat{R}$ by local duality.) Q.E.D.

(4.7) REMARK. If $R$ is an Artin local ring, a canonical module for $R$ is precisely the same as an injective hull for $K$ as an $R$-module.

Recall that a (not necessarily local) Noetherian ring $R$ is generically Gorenstein if its total quotient ring is Gorenstein. We need the following useful though elementary:

(4.8a) FACT. Let $R$ be a generically Gorenstein Cohen-Macaulay local ring which possesses a canonical module $E$. Then $R$ and $E$ can each be embedded in the other. Moreover, $E$ is isomorphic as an $R$-module with an ideal of $R$ which may be taken to be $R$ precisely if $R$ is Gorenstein and which otherwise has pure height one.

Proof. Let $T$ denote the multiplicative system of nonzerodivisors in $R ; T=R-\cup_{i=1}^{m} p_{i}$, where $p_{1}, \ldots, p_{m}$ are the minimal primes of $R . T^{-1} R$ is a zero-dimensional Gorenstein ring and $T^{-1} R \cong \prod_{i=1}^{m} R_{p_{i}} \cdot T^{-1} E$ $\cong \prod_{i=1}^{m} E_{p_{i}}$, and since each $R_{p_{i}}$ is Gorenstein and $E_{p_{i}}$ is a canonical module 
for $R_{p_{i}}$, we have $E_{p_{i}} \cong R_{p_{i}}$. Hence, $T^{-1} R \cong T^{-1} E$. Now, we have injections $R \hookrightarrow T^{-1} R, E \hookrightarrow T^{p_{i}-1} E$. Hence, the isomorphism $T^{-1} R \cong T^{-1} E$ provides injections $R \hookrightarrow T^{-1} E, E \hookrightarrow T^{-1} R$. Since $R$ and $E$ are $R$-modules of finite type, we may choose nonzerodivisors $a, b \in R$ such that the image of $a R(\cong R)$ in $T^{-1} E$ is contained in $E$, and the image of $b E(\cong E)$ in $T^{-1} R$ is contained in $R$. Thus, we have injections $R \hookrightarrow E$ and $E \hookrightarrow R$. Let $I$ be an ideal of $R$ such that $I \cong E$. Let $P$ be an associated prime of $R$ and let $A=R_{P}$. Then $I A$ is a canonical module for $A$, and we cannot have height $P \geqslant 2$, for then $\operatorname{depth}_{A} A \geqslant 2, \operatorname{depth}_{A} I A \geqslant 2$, and so $\operatorname{depth}_{A} A / I A \geqslant 1$, while $P$ $\in \operatorname{Ass}(R / I) \Rightarrow \operatorname{depth}_{A} A / I A=0$. Q.E.D.

(4.8b) Remarks. If $R$ is Cohen-Macaulay and has a canonical module, then if $R$ is generically Gorenstein, $R$ is approximately Gorenstein. For the canonical module $E$ always has S.C.I.: if $x_{1}, \ldots, x_{n}$ is a system of parameters for $R$, then $\left(x_{1}^{N}, \ldots, x_{n}^{N}\right) E$ is irreducible in $E$. If $R$ is generically Gorenstein, we can embed $R \hookrightarrow E$, and then $R$ has S.C.I., i.e., $R$ is approximately Gorenstein. See Example (5.4).

We are almost ready to prove the necessity of condition $(*)$ for a module to have S.C.I. First, we want to make some observations.

(4.9) Discussion. Let $R$ be Noetherian (not necessarily local). If $P$ is a prime of $R$, we write $\mu_{i}(P, M)$ for

$$
\operatorname{dim}_{\kappa(P)} \operatorname{Ext}_{R_{P}}^{i}\left(\kappa(P), M_{P}\right),
$$

where $\kappa(P)=R_{P} / P R_{P}: \mu_{i}(P, M)$ is the $i$ th Bass number (and can also be characterized as the number of copies of the injective hull over $R$ (or over $R_{P}$ ) of $\kappa(P)$ occurring in the $i$ th term in a minimal injective resolution of $M[1]$. Here, we shall be concerned solely with the numbers $\mu_{0}(P, M)$, or, briefly, $\mu(P, M)$. We note the following trivial facts (the proofs are left to the reader):

(4.10) Lemma. Let $R$ be Noetherian (not necessarily local).

(a) $\mu(P, M)=\mu\left(P R_{P}, M_{P}\right)$.

(b) $\mu(P, M)>0 \Leftrightarrow P \in \operatorname{Ass}(M)$.

(c) $\mu(P, M)$ is the torsion-free rank of any maximal $(R / P)$-torsion-free submodule of $M$.

(d) $\mu(P, M)$ is the rank of any maximal $(R / P)$-free submodule of $M$.

(e) If $P$ is minimal in $R, R_{P}$ is Gorenstein if and only if $\mu(P, R)=1$.

(4.11) THEOREM. If $M$ has S.C.I. and $P$ is a prime of $R$ such that $\operatorname{dim}(R / P)$ $=1$, then $\mu(P, M) \leqslant 1$. In other words, $(R / P) \oplus(R / P)$ cannot be embedded in $M$.

Proof. If not, we can choose a prime $Q$ (of $\hat{R}) \in \operatorname{Ass}(\hat{R} / P)$ such that $\operatorname{dim} \hat{R} / Q=1$. Then we have an embedding $\hat{R} / Q \hookrightarrow \hat{R} / \hat{P}$ and hence an embedding 


$$
(\hat{R} / Q) \oplus(\hat{R} / Q) \hookrightarrow(\hat{R} / \hat{P}) \oplus(\hat{R} / \hat{P}) \cong((R / P) \oplus(R / P)) \hookrightarrow \hat{M} .
$$

Thus, there is no loss of generality in assuming that $R$ is complete. By (4.3), $(R / P) \oplus(R / P)$ will itself have S.C.I.: by (4.1c), we may replace $R$ by $R / P$.

Thus, it suffices to show that if $R$ is a complete one-dimensional local domain, then $R \oplus R$ does not have S.C.I.

Let $u \in m-\{0\}$. Since $\operatorname{dim} R=1$, we can choose $N$ such that $m^{N} \subset u R$. We shall show that if $M^{\prime}$ is cofinite and irreducible in $R^{2}$, then $M^{\prime} \nsubseteq m^{N}\left(R^{2}\right)$. To see this, let $V=R^{2} / M^{\prime}$. Since $V$ is an essential extension of $K$ (of finite length), we have an injection $V \hookrightarrow W$, where $W$ is the injective hull of $K$. By (4.6), if $I$ denotes an ideal $R$ which is a canonical module for $R$ (cf. (4.8)), then $W=\operatorname{proj}_{\lim _{t}} I / u^{t} I$, and since $V$ has finite length it follows that for some $t$ we have an injection $V \rightarrow I / u^{t} I$. We then have the following commutative diagram in which the sequences are exact:

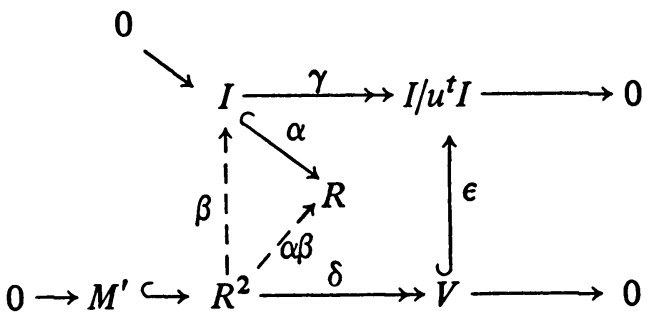

where $\alpha$ is simply the inclusion of $I$ in $R$. We can choose $\beta$ (and then, uniquely, $\alpha \beta$ ) so that the diagram commutes, since $\gamma$ is surjective and $R^{2}$ is free. Let $a$, $b$ be the images of the free generators for $R^{2}$ in $R$ (under $\alpha \beta$ ). We may choose a largest possible nonnegative integer $h$ such that $a=u^{h} a^{\prime}, b=u^{h} b^{\prime}$ with $a^{\prime}, b^{\prime} \in R$. Since $h$ is as large as possible, either $a^{\prime} \notin u R$ or $b^{\prime} \notin u R$. Hence $\left(b^{\prime},-a^{\prime}\right) \notin m^{N} R^{2}$ (for $\left.m^{N} \subset u R\right)$. But $\alpha \beta\left(\left(b^{\prime},-a^{\prime}\right)\right)=b^{\prime} a-a^{\prime} b=0$. Since $\alpha$ is injective, $\beta\left(\left(b^{\prime},-a^{\prime}\right)\right)=0$ and so $\gamma \beta\left(\left(b^{\prime},-a^{\prime}\right)\right)=0$. But $\gamma \beta=\varepsilon \delta$, so that $\varepsilon \delta\left(\left(b^{\prime},-a^{\prime}\right)\right)=0$, and so, since $\varepsilon$ is injective, $\delta\left(\left(b^{\prime},-a^{\prime}\right)\right)=0$, i.e., $\left(b^{\prime},-a^{\prime}\right)$ $\in M^{\prime}$. Thus, $M^{\prime} \nsubseteq m^{N} R^{2}$. Q.E.D.

(4.4) and (4.11) together yield the necessity of the condition (*) for an $M$ with $\operatorname{dim} M>0$ to have S.C.I.: part of the statement of Theorem (1.12).

We now have enough information to prove the first statement in Proposition (1.13).

(4.12) Proposition. Suppose that every $M$ with $\operatorname{dim} M>0$ which satisfies (*) has S.C.I. Then $R$ is an E-ring.

Proof. Suppose, to the contrary, that $R$ is not an $E$-ring. There are two possibilities. One is that for some prime $P$ of $R, \operatorname{dim} R / P \geqslant 2$ and $\operatorname{Ass}(R / P)^{\hat{n}}$ contains primes of coheight 1 . Let $Q \in \operatorname{Ass}(R / P)^{\wedge}$ be such that $\operatorname{dim} \hat{R} / Q=1$. 
Then $(R / P) \oplus(R / P)$ satisfies $(*)$, but its completion has $\hat{R} / Q \oplus \hat{R} / Q$ as a submodule and does not. Hence, by $(4.11),((R / P) \oplus(R / P))^{\wedge}$ does not have S.C.I., and so neither does $(R / P) \oplus(R / P)$.

The second possibility is that for some prime $P$ of $R$ with $\operatorname{dim}(R / P)=1$, the ring $(R-P)^{-1}(R / P)^{\hat{n}}$ is not Gorenstein. This means that for some minimal prime $Q$ of $\hat{P},\left((R / P)^{\hat{n}}\right)_{Q}$ is not Gorenstein. Since this ring is zerodimensional, this says (cf. (4.10e)) that $\mu\left(Q / \hat{P},(R / P)^{\hat{}}\right) \geqslant 2$, i.e., $(\hat{R} / Q)$ $\oplus(\hat{R} / Q)$ is embeddable in $(R / P)^{\hat{n}}$. Thus, by $(4.11),(R / P)^{\hat{n}}$ does not have S.C.I. But $R / P$ satisfies (*). Thus, in either case, we have a contradiction. Q.E.D.

Our remaining objective is the result that if $R$ is an $E$-ring and $\operatorname{dim} M \geqslant 1$, then (*) is sufficient for $M$ to have S.C.I. We need a refined version of primary decomposition for modules [4, Chapter I, §B].

First, recall that if $P$ is a prime of $R$ (not necessarily local) then $M$ is " $P$ coprimary" if, equivalently, (i) $\operatorname{Ass}(M)=\{P\}$ or (ii) $\operatorname{Rad} \operatorname{Ann} M=P$, and for every $r \in R$ either $r$ is not a zerodivisor on $M$ or $r \in P$ (in which case $r$ is nilpotent on $M$ ).

(4.13) Lemma. Let $R$ be a (not necessarily local) Noetherian ring and $M$ an $R$ module of finite type. Let $\operatorname{Ass}(M)=\left\{P_{1}, \ldots, P_{h}\right\}$ and let $\nu_{i}=\mu\left(P_{i}, M\right), 1 \leqslant i$ $\leqslant h$. For each $i$, let $M_{i}$ be a copy of $\left(R / P_{i}\right)^{v_{i}}$ in $M$.

(a) Let $M^{*}=\sum_{i=1}^{h} M_{i}$. Then the sum is direct. Moreover, $M$ is an essential extension of $M^{*}$.

Since $M_{i} \cap \sum_{j \neq i} M_{j}=0$, for each $i$ we may extend $\sum_{j \neq i} M_{j}$ to a submodule $E_{i}$ of $M$ maximal with respect to being disjoint from $M_{i}$. Let $M^{\prime}$ $=\sum \oplus_{i=1}^{h}\left(M / E_{i}\right)=\prod_{i=1}^{h}\left(M / E_{i}\right)$. The maps $M \rightarrow M / E_{i}$ induce a map $M$ $\rightarrow M^{\prime}$.

(b) For each $i, M / E_{i}$ is an essential extension of the image of $M_{i}$, and, hence, $\operatorname{Ass}\left(M / E_{i}\right)=\left\{P_{i}\right\}$, i.e., $M / E_{i}$ is $P_{i}$-coprimary and $E_{i}$ is $P_{i}$-primary. Moreover, $\mu\left(P_{i}, M / E_{i}\right)=\nu_{i}$.

(c) The map $M \rightarrow M^{\prime}$ is injective.

(d) $\cap_{i=1}^{h} E_{i}=0$; in fact, this is an irredundant primary decomposition of 0 in M.

Proof. (a) If $V_{1}, V_{2}$ are submodules of $M$ such that $\operatorname{Ass}\left(V_{1}\right) \cap \operatorname{Ass}\left(V_{2}\right)=\varnothing$, then $V_{1} \cap V_{2}=0$ and $V_{1}+V_{2}=V_{1} \oplus V_{2}$, for $\operatorname{Ass}\left(V_{1} \cap V_{2}\right) \subset \operatorname{Ass}\left(V_{1}\right)$ $\cap \operatorname{Ass}\left(V_{2}\right)$. By induction, if $V_{1}, \ldots, V_{t}$ are submodules of $M$ and the sets $\operatorname{Ass}\left(V_{i}\right)$ are pairwise disjoint, $V_{1}+\cdots+V_{t}=V_{1} \oplus \cdots \oplus V_{t}$. Since $\operatorname{Ass}\left(M_{i}\right)$ $=\left\{P_{i}\right\}, M^{*}=\sum_{i=1}^{h} M_{i}=\sum \oplus_{i=1}^{h} M_{i}$. To see that the extension $M^{*} \rightarrow M$ is essential, let $w \in M-\{0\}$ be given: we must show that $R w \cap M^{*} \neq 0$. First, we replace $w$ by a multiple whose annihilator is prime. Thus, we may assume $\operatorname{Ann}_{R} w=P_{i}$ for a certain $i$, and $R w \cong R / P_{i}$. But then, $R w \cap M^{*}=0$ 
$\Rightarrow(R w) \cap M_{i}=0$ and then $R w+M_{i} \cong R w \oplus M_{i} \cong\left(R / P_{i}\right)^{\nu_{i}+1}$, contradicting the definition of $\nu_{i}$. This proves (a).

(b) Since $E_{i} \cap M_{i}=0$, we have an injection $M_{i} \rightarrow M / E_{i}$ : the maximality of $E_{i}$ says precisely that this is an essential extension. The rest is straightforward.

(c) Since $M$ is an essential extension of its submodule $M^{*}=\sum \oplus_{i} M_{i}$, it suffices to show that the restriction $M^{*} \rightarrow M^{\prime}$ is injective, i.e., that $\phi: \sum \oplus_{i} M_{i}$ $\rightarrow \Pi_{i} M / E_{i}$ is injective. If $j \neq i, M_{j} \subset E_{i}$ by construction of $E_{i}$, so that each summand $M_{i}$ maps to 0 in all coordinates except the $i$ th. Thus, writing $\Pi_{i}\left(M / E_{i}\right)=\sum \oplus_{i}\left(M / E_{i}\right)$ we see that $\phi$ is the direct sum of the maps $M_{i} \rightarrow M / E_{i}$, which we already know are injective.

(d) The injectivity of $M \rightarrow \Pi_{i}\left(M / E_{i}\right)$ says precisely that $\cap_{i} E_{i}=0$, while from (b), $E_{i}$ is $P_{i}$-primary. Q.E.D.

(4.14) LemMa. Let $R$ be a (not necessarily local) Noetherian ring, $P$ a prime ideal of $R$, and $M$ a P-coprimary module with $\mu(P, M)=\nu$. Then there exist $\nu$ $P$-coprimary modules $M_{1}, \ldots, M_{\nu}$ such that $\mu\left(P, M_{i}\right)=1,1 \leqslant i \leqslant \nu$, and $M$ can be embedded in $\sum \bigoplus_{i=1}^{\nu} M_{i}$.

Proof. We may replace $R$ by $R /$ Ann $M$ without loss of generality, and so assume that $P$ is the unique minimal prime of $R$. Now, suppose we can solve the problem over $R_{P}$, and embed $M_{P} \hookrightarrow \sum \oplus_{i=1}^{\nu} M_{i}^{*}$, where the $M_{i}^{*}$ are suitable $R_{P}$-modules. Each $M_{i}^{*}$ has the form $\left(M_{i}\right)_{P}$ for a suitable $R$-module of finite type $M_{i}$, and $M_{i}$ may be chosen $P$-coprimary as well. Since $M$ is of finite type, for a suitable $s \in R-P$ the image of $s M \cong M$ in $\sum \oplus_{i=1}^{\nu} M_{i}^{*}$ $=\left(\sum \oplus_{i=1}^{\nu} M_{i}\right)_{P}$ will be contained in $\sum \oplus_{i=1}^{\nu} M_{i}$, and we are done.

Thus, we may assume that $(R, P)$ is a zero-dimensional local ring, say with residue class field $K=R / P$, and, as in (4.13a), $M$ is an essential extension of $K^{\nu} \subset M$. Now, let $M_{1}^{*}, \ldots, M_{\nu}^{*}$ each be the injective hull of $K$ as an $R$ module. The maps $K \hookrightarrow M_{i}^{*}$ give rise to an injection $K^{\nu} \rightarrow \sum \oplus_{i=1}^{\nu} M_{i}^{*}$ which, since $\sum \bigoplus_{i=1}^{\nu} M_{i}^{*}$ is $R$-injective, extends to a map $M \rightarrow \sum \bigoplus_{i=1}^{\nu} M_{i}^{*}$; moreover, since $K^{\nu} \subset M$ is essential, this map is injective. Q.E.D.

(4.15) Proposition. Let $R$ be a (not necessarily local) Noetherian ring and let $M$ be an $R$-module of finite type with $\operatorname{Ass}(M)=\left\{P_{1}, \ldots, P_{h}\right\}$ and $\mu\left(P_{i}, M\right)=\nu_{i}$. Then $M$ can be embedded in a direct sum of $\sum_{i=1}^{h} \nu_{i} R$-modules $\sum \oplus_{1 \leqslant i \leqslant h ; 1 \leqslant j \leqslant \nu} M_{i j}$, where $M_{i j}$ is a $P_{i}$-coprimary module with $\mu\left(P_{i}, M_{i j}\right)=1,1$ $\leqslant i \leqslant h, 1 \leqslant j \leqslant \nu_{i}$.

Proof. By (4.13) we can embed $M \rightarrow \sum \oplus_{i=1}^{h} M / E_{i}$ where $M / E_{i}$ is $P_{i}$ coprimary and $\mu\left(P_{i}, M / E_{i}\right)=\nu_{i}$, and by (4.14) we can embed $M / E_{i}$ in a direct $\operatorname{sum} \sum \oplus_{j=1}^{v_{i}} M_{i j}$ where each $M_{i j}$ is $P_{i}$-coprimary and $\mu\left(P_{i}, M_{i j}\right)=1$. Q.E.D.

We need one final preparatory lemma in order to prove Theorem 1.12.

(4.16) LemMa. Let $R$ be an E-ring and let $M$ be a module which satisfies 
condition (*). Then $\hat{M}$ satisfies condition (*) as an $\hat{R}$-module.

Proof. Since depth $M=1$, depth $\hat{M}=1$. Thus, the problem is to show that if $Q$ is a prime of $\hat{R}$ with $(\hat{R} / Q)=1$, then $\mu(Q, \hat{M}) \leqslant 1$. By [21, Proposition 15, p. IV-25], $\operatorname{Ass}_{R}(\hat{M})=\bigcup_{i=1}^{h} \operatorname{Ass}_{R}\left(R / P_{i}\right)^{\hat{h}}$. Since $R$ is an $E$ ring, if $\operatorname{dim}\left(R / P_{i}\right) \geqslant 2$, no prime in $\operatorname{Ass}_{R}(R / P)^{\wedge}$ has coheight 1 . Thus, we need only show that if $P \in \operatorname{Ass}(M)$ and coheight $P=1$, then if $Q \in \operatorname{Ass}_{R}(R / P)^{\wedge}$ then $\mu(Q, \hat{M}) \leqslant 1$. But if $Q \in \operatorname{Ass}_{\hat{R}}(R / P)^{\hat{n}}$ then $Q \supset P \hat{R}$, i.e., there is a surjection $(R / P)^{\hat{\imath}} \rightarrow \hat{R} / Q$, which in turn yields an injection

$\operatorname{Hom}_{\hat{R}}(\hat{R} / Q, \hat{M}) \hookrightarrow \operatorname{Hom}_{R}\left((R / P)^{\wedge}, \hat{M}\right) \cong\left(\operatorname{Hom}_{R}(R / P, M)\right)^{\wedge} \cong\left(\operatorname{Ann}_{M} P\right)^{\wedge}$.

Since $\operatorname{dim} R / P=1, \operatorname{Ann}_{M} P$ is a torsion-free $(R / P)$-module: otherwise, $m$ $\in \operatorname{Ass}\left(\operatorname{Ann}_{M} P\right) \subset \operatorname{Ass}(M), \quad$ a contradiction. Since $\mu(P, M) \leqslant 1$, rank $\operatorname{Ann}_{M} P \leqslant 1$, and hence $\mathrm{Ann}_{M} P$ is embeddable in $R / P$. Thus, we have $\left(\operatorname{Ann}_{M} P\right)^{\wedge} \hookrightarrow(R / P)^{\wedge}$ and so $\operatorname{Hom}_{R}(\hat{R} / Q, \hat{M}) \hookrightarrow(R / P)^{\wedge}$. Since $R$ is an $E$ ring if we localize $(R / P)^{\wedge}$ at $Q$ it becomes a zero-dimensional Gorenstein ring, and we have an injection:

$$
\operatorname{Hom}_{R_{Q}}\left(\hat{R}_{Q} / Q \hat{R}_{Q}, \hat{M}_{Q}\right) \hookrightarrow\left((R / P)^{\hat{n}}\right)_{Q}
$$

It follows that $\mu(Q, \hat{M})=\mu\left(Q R_{Q}, \hat{M}_{Q}\right) \leqslant \mu\left(Q,(R / P)_{Q}\right)=1$. Q.E.D.

We are now ready to prove the hardest part: that $(*)$ is sufficient for having S.C.I. when $R$ is an $E$-ring. Of course, we have already done a good deal of the work.

(4.17) Theorem. Let $R$ be an E-ring and let $M$ be an $R$-module with $\operatorname{dim} M>0$. Suppose that $M$ satisfies condition (*). Then $M$ has small cofinite irreducibles.

Proof. We proceed by induction on $n=\operatorname{dim} M=\operatorname{dim}(R / \operatorname{Ann} M)$. By (4.16) we may assume that $R$ is complete. By the induction hypothesis we know the result for all modules $M^{\prime}$ with $\operatorname{dim} M^{\prime}<n$, even if the base ring is an $E$-ring and not necessarily complete.

We first consider the case in which $\operatorname{dim} M=1$. Then all the primes in $\operatorname{Ass}(M)$ have coheight one. Since $R$ is complete, we can map a complete regular local ring onto it. Then, by (4.1c), we may assume that $R$ is complete regular. Let $I=\operatorname{Ann}(M)$. Then $I$ will have pure height $(\operatorname{dim} R)-1$, and hence, since $R$ is regular and, in particular, Cohen-Macaulay, $I$ will contain an $R$-sequence of length $(\operatorname{dim} R)-1$. But $R$ regular $\Rightarrow R$ Gorenstein, and so we may divide out by this $R$-sequence ((4.1c) again) and replace $R$ by a onedimensional Gorenstein ring.

Thus, if $\operatorname{dim} M=1$ we may assume that $\operatorname{dim} R=1$ and that $R$ is a complete Gorenstein local ring. We have that for each minimal prime $P$ of $R$, 
$\mu(P, M) \leqslant 1$. But then $M$ can be embedded in $R$. To see this, note that since depth $M=1=\operatorname{dim} R, M$ is a Cohen-Macaulay module and so every nonzerodivisor in $R$ is a nonzerodivisor on $M$. Let $T$ be the multiplicative system of nonzerodivisors of $R$. Then $R \hookrightarrow T^{-1} R, M \hookrightarrow T^{-1} M$, and, as usual, it will suffice to embed $T^{-1} M \hookrightarrow T^{-1} R$. But $T^{-1} R$ is a product of zero-dimensional Gorenstein rings and $T^{-1} M \cong \Pi_{P} M_{P}$ where $P$ runs through the minimal primes of $R . \mu(P, M) \leqslant 1 \mu\left(P R_{P}, M_{P}\right) \leqslant 1 \Rightarrow M_{P}=0$ or else $M_{P}$ is an essential extension of $\kappa(P)=R_{P} / P R_{P}$. Since $R_{P}$ is Gorenstein, it is the injective hull (maximal essential extension) of $\kappa(P)$, so that for each $P$ we have an embedding $M_{P} \hookrightarrow R_{P}$ and hence an embedding $T^{-1} M \cong \Pi_{P} M_{P}$ $\hookrightarrow \Pi_{P} R_{P}=T^{-1} R$, as required.

Since $R$ is Gorenstein, it is approximately Gorenstein and has S.C.I. and, hence, so does its submodule $M$.

This completes the proof for the case $n=1$.

We now suppose $n>1$. We are retaining the assumption that $R$ is complete and that the result holds for any $R^{\prime}, M^{\prime}$ if $R^{\prime}$ is an $E$-ring and $\operatorname{dim} M^{\prime}<n$. Let $\operatorname{Ass}(M)=\left\{P_{1}, \ldots, P_{h}\right\}$ and embed $M \hookrightarrow \sum \oplus_{1 \leqslant i \leqslant h ; 1 \leqslant j \leqslant \nu_{i}} M_{i j}$ precisely as in Proposition (4.15): $M_{i j}$ is $P_{i}$-coprimary, $\nu_{i}=\mu\left(P_{i}, M\right)$, and $\mu\left(P_{i}, M_{i j}\right)=1,1$ $\leqslant i \leqslant h, 1 \leqslant j \leqslant \nu_{i}$.

Then we can select $\sum_{i=1}^{h} v_{i}$ distinct primes $P_{i j}$ with the following properties:

(1) For each $i, j, \operatorname{dim} R / P_{i j}=1$.

(2) If $\operatorname{dim}\left(R / P_{i}\right)=1$ (this implies $\nu_{i}=1$, since $M$ satisfies $\left.(*)\right)$, then $P_{i j}=P_{i}$.

(3) For each $i, j, P_{i j} \supset P_{i}$.

Note that in order to select these primes we must have $m \notin \operatorname{Ass}(M)$, and we also must have $\mu\left(P_{i}, M\right)=\nu_{i}=1$ if $\mathrm{ht}\left(R / P_{i}\right)=1$. (To select the $P_{i j}$ first pick $P_{i j}=P_{i}$ for those $i$ such that $\operatorname{dim} R / P_{i}=1$. The other $P_{i j}$ may be selected one at a time: given $i, j$, where $\operatorname{dim} R / P_{i} \geqslant 2$, let $P_{i j}$ be any prime which contains $P_{i}$, has coheight 1 in $R$, and which has not already been used. This is possible, since $R / P_{i}$ has infinitely many primes of coheight 1.)

By (4.3), we may replace $M$ by $\Sigma \oplus_{i j} M_{i j}$ : it will suffice to show that $M=\sum \oplus_{i j} M_{i j}$ has S.C.I.

Let an integer $N>0$ be given. By (4.2) it will suffice to show that there is a module $M_{N} \subset m^{N} M$ such that $M / M_{N}$ has S.C.I. Let $M_{i j}^{*}$ be the localization of $M_{i j}$ at $P_{i j}$. Since $M_{i j}$ is $P_{i}$-coprimary and $P_{i} \subset P_{i j}, M_{i j} \subset M_{i j}^{*}$. Let $M_{i j k}$ $=P_{i j}^{k} M_{i j}^{*} \cap M_{i j}$. Since $\cap_{k} P_{i j}^{k} M_{i j}^{*}=0, \cap_{k} M_{i j k}=0$. Since $M_{i j}$ is a module over a complete local ring, and $M_{i j k}$ is a decreasing sequence of submodules whose intersection is 0 , we can choose an integer $k=N_{i j}$ such that $M_{i j N_{i j}}$ $\subset m^{N} M_{i j}$.

Now, since $R$ is complete, if $R_{i j}$ denotes the localization of $R$ at $P_{i j}$, then $R_{i j}$ is excellent and, in particular, an $E$-ring. Since $M_{i j}$ is $P_{i}$-coprimary and $\mu\left(P_{i}, M_{i j}\right)=1$, we have that $M_{i j}^{*}$ is $P_{i} R_{i j}$-coprimary and $\mu\left(P_{i} R_{i j}, M_{i j}^{*}\right)=1$. 
Hence, $M_{i j}^{*}$ satisfies $(*)$ over $R_{i j}$. But $\operatorname{dim} M_{i j}^{*}<\operatorname{dim} M_{i j} \leqslant \operatorname{dim} M$; hence, $M_{i j}^{*}$ has S.C.I. as an $R_{i j}$-module. It follows that we can choose an $R_{i j}$ submodule $E_{i j}^{*}$ of $M_{i j}^{*}$ which is cofinite, irreducible, and such that $E_{i j}^{*}$ $\subset\left(P_{i j}\right)^{N_{i j}} M_{i j}^{*}$. Let $E_{i j}=E_{i j}^{*} \cap M_{i j}$. By the choice of $N_{i j}, E_{i j} \subset m^{N} M_{i j}$. Moreover, it is easy to see that $M_{i j} / E_{i j}$ is $P_{i j}$-coprimary and $\mu\left(P_{i j}, M_{i j} / E_{i j}\right)=1$. Thus, we may let $M_{N}=\sum \bigoplus_{i j} E_{i j}$, for then $M_{N} \subset m^{N} M$ and $M / M_{N}$ $=\sum \bigoplus_{i j}\left(M_{i j} / E_{i j}\right)$ has S.C.I. by the case $n=1$ (of course, we are using here the fact that the $P_{i j}$ are all distinct). Q.E.D.

5. Approximately Gorenstein rings, cyclic purity, and purity, revisited. We have now done all the real work, and we can reap a harvest of corollaries.

(5.1) Corollary. Let $R$ be a Noetherian ring. The condition that $R$ be approximately Gorenstein is local on the maximal ideals of $R$, i.e., $R$ is approximately Gorenstein if and only if for every maximal ideal $m$ of $R, R_{m}$ is approximately Gorenstein.

Proof. This is immediate from the definition. Q.E.D.

(5.2) THEOREM. Let $R$ be a Noetherian ring. If $R$ is an E-ring (in particular, if $R$ is a locally excellent Noetherian ring, especially, a complete local ring), then $R$ is approximately Gorenstein if and only if the following two conditions are satisfied:

(1) If $m$ is a maximal ideal of $R$ and $m \in \operatorname{Ass}(R)$, then $R_{m}$ is a zerodimensional Gorenstein ring.

(2) If $P \in \operatorname{Ass}(R)$ has coheight 1 in a maximal $m$ of $R$, then $\mu(P, R)=1$, i.e., $R / P \oplus R / P$ is not embeddable in $R$.

Hence, for an arbitrary Noetherian ring $R, R$ is approximately Gorenstein if and only if for each maximal ideal $m$ of $R$, either $R_{m}$ is a zero-dimensional Gorenstein ring or else $m \notin \operatorname{Ass}(R)$ and for each $Q \in \operatorname{Ass}\left(\hat{R}_{m}\right)$, if $\operatorname{dim}\left(\hat{R}_{m} / Q\right)$ $=1$ then $\mu\left(Q, \hat{R}_{m}\right)=1$.

Proof. The result is immediate from (5.1), (2.2c), (1.5), (1.11c), and (1.12). Q.E.D.

Of course, (1.6) is just the special case where $R$ is complete local and $\operatorname{dim} R \geqslant 1$.

(5.3) Proof of (1.7). Since (1) $\Rightarrow(2)$, it suffices to show that either (2) or (3) implies the condition of (5.2). If (3) holds then the condition of (5.2) holds vacuously, because $\operatorname{Ass}(R)$ has no elements of coheight $\leqslant 1$ in a maximal ideal. (2) implies that all primes $P$ in $\operatorname{Ass}(P)$ are minimal, and that for each $P \in \operatorname{Ass}(R), \mu(P, R)=\mu\left(P R_{P}, R_{P}\right)=1$, since $R_{P}$ is Gorenstein. Q.E.D.

(5.4) EXAMPLE. Let $r$ be an integer $\geqslant 2$. By Proposition 3.1 of [5], there is a local domain $A$ of dimension 1 (thus, $A$ is Cohen-Macaulay) such that $\hat{A}$ possesses a unique minimal prime $P$ such that 
(1) $P^{2}=0$

(2) $P \cong(\hat{A} / P)^{r}$.

Thus, $\hat{A}$ is not generically Gorenstein, and, hence, neither is $A$. We can therefore embed $A \rightarrow S$, ideally closed, such that $A \rightarrow S$ is not pure. Since $S$ is the direct limit of its finitely generated $A$-subalgebras, we may even take $S$ to be finitely generated as an $A$-algebra. Moreover, $A$ has equal characteristic 0 . Thus, there is a one-dimensional (Cohen-Macaulay) local domain $A$ of equal characteristic 0 and a finitely generated extension algebra $A \rightarrow S$ such that $A \rightarrow S$ is ideally closed but not pure.

The fact that $A$ is not approximately Gorenstein is closely related to the fact that it has no canonical module; if $A$ had a canonical module, it would be embeddable in it, and, hence, approximately Gorenstein. See (4.8b).

We conclude this section with two more corollaries of our main results.

(5.5) Proposition. Let $R$ be an approximately Gorenstein Noetherian ring, for example, a reduced excellent Noetherian ring, and let $S$ be a module-finite extension algebra. Suppose that $I S \cap R=I$ for every ideal $I$ of $R$. Then $R$ is a direct summand of $S$ as an $R$-module.

Proof. The hypothesis guarantees that $R$ is cyclically pure in $S$, and, since $R$ is approximately Gorenstein, that $R$ is pure in $S$. But then, since $S / R$ is a finitely generated ( $\Rightarrow$ finitely presented) $R$-module, $R$ is a direct summand of S. Q.E.D.

(5.6) Proposition. Let $R$ be any Noetherian ring and let $x, y$ be analytic indeterminates over $R$. Then $R[x, y]$ and $R[[x, y]]$ are approximately Gorenstein.

Moreover, if $R \hookrightarrow U$ is any algebra extension (or even an $R$-module extension) the following five conditions are equivalent:

(i) $R$ is pure in $U$.

(ii) $R[x, y]$ is cyclically pure in $U[x, y]$.

(iii) $R[[x, y]]$ is cyclically pure in $U[[x, y]]$.

(iv) $R[x, y]$ is pure in $U[x, y]$.

(v) $R[[x, y]]$ is pure in $U[[x, y]]$.

Proof. Let $Q$ be a maximal ideal in $S=R[x, y]$ (respectively, $S=$ $R[[x, y]])$ lying over say, $P$, in $R$. We must show that $S_{Q}$ is approximately Gorenstein for each $Q$, or, equivalently, that $\hat{S}_{Q}$ is approximately Gorenstein for each $Q$. (Note that in the case $S=R[[x, y]], P$ must be maximal in $R$ and we must have $Q=(P, x, y) S$.) Let $T=\hat{R}_{P}[x, y]$ (resp., $\left.\hat{R}_{P}[[x, y]]\right)$ and let $m=Q T$. Then $\hat{T}_{m}=\hat{S}_{Q}$, so that it suffices to show that if $A\left(\right.$ let $\left.A=\hat{R}_{P}\right)$ is a complete local ring, then $B=A[x, y]$ (resp. $B=A[[x, y]]$ ) is approximately Gorenstein. But $\operatorname{Ass}_{B} B=\left\{p B: p \in \operatorname{Ass}_{A}(A)\right\}$ and if $p \in \operatorname{Ass} A, B / p B$ $\cong(A / p)[x, y]($ resp. $(A / p)[[x, y]])$ has the property that every maximal ideal has 
height at least two. Since $B$ is excellent, by Theorem (1.7) it is approximately Gorenstein.

We now prove the equivalence of (i)-(v). From our point of view, the interest is in the implications (ii) $\Rightarrow$ (i) and (iii) $\Rightarrow$ (i). In fact, (i) $\Rightarrow$ (iv) is trivial (since $R[x, y] \rightarrow S[x, y]$ arises by tensoring $R \rightarrow S$ with $R[x, y]$ over $R$ ), and (i) $\Rightarrow$ (v) is known (see [2]). Moreover, (v) $\Rightarrow$ (iii) and (iv) $\Rightarrow$ (ii) are quite evident.

To see why (ii) (or (iii)) implies (i) let $S=R[x, y]$ (resp., $R[[x, y]]$ ) and $T=U[x, y]$ (resp., $U[[x, y]]$ ). Since $S$ is approximately Gorenstein, the cyclic purity of $S \rightarrow T$ implies the purity of $S \rightarrow T$. Now $R \rightarrow S$ is pure (in fact, $R$ is an $R$-algebra retract of $S$ via a homomorphism which kills $(x, y) S)$, and so $R \rightarrow T$ is pure. Since $U$ is an $R$-algebra retract of $T, R \rightarrow U$ is pure as well. Q.E.D.

(5.7) Remark. It is well known (see [2], [4]), that if $R\left[x_{1}, \ldots, x_{n}\right]$ $\rightarrow S\left[x_{1}, \ldots, x_{n}\right]$ is cyclically pure for every $n$, then $R$ is pure in $S$, even if $R$ is not Noetherian. The interesting point in (ii) $\Rightarrow$ (i) is that if $R$ is Noetherian, we need only let $n=2$.

6. Adjunction of analytic indeterminates fails to preserve purity in the nonNoetherian case. In [2] it is shown that if $R$ is Noetherian and $R \hookrightarrow S$ is pure, then $R[[t]] \hookrightarrow S[[t]]$ is pure. In this section we construct a counterexample when $R$ is not Noetherian. In fact, we prove the following:

(6.1) Proposition-Example. Let $K$ be any commutative ring, e.g., a field. Then there exists a countably generated $K$-algebra $R$, and a countably generated ring extension $R \hookrightarrow S$ such that

(1) $R \hookrightarrow S$ is pure.

(2) $R[[t]] \rightarrow S[[t]]$ is not even cyclically pure: in fact there is a principal ideal of $R[[t]]$ which is not contracted from $S[[t]]$.

Proof. We construct $R, S$ explicitly as follows. Let $\left\{X_{j}, Z_{i j}, W_{j}: i, j\right\}$, where $i, j$ are nonnegative integers and $i \leqslant j$, be countably many indeterminates. Let $T=K\left[X_{j}, Z_{i, j}: i \leqslant j\right]$. In $T$, if $j^{\prime}, j \geqslant n \geqslant 0$, let

$$
p\left(j, j^{\prime}, n\right)=\sum_{i=0}^{n} X_{i}\left(Z_{n-i, j}-Z_{n-i, j^{\prime}}\right) .
$$

Let $I$ be the ideal of $T$ generated by the elements $p\left(j, j^{\prime}, n\right)$ and let $R=T / I$. We write $x_{i}, z_{i j}$ for the images of $X_{i}, Z_{i j}$, respectively, in $R$. Thus,

$$
R=K\left[x_{i}, z_{i j}: i \leqslant j\right]
$$

and if we let $y_{n}=\sum_{i=0}^{n} x_{i} z_{n-i, j}$ for any $j \geqslant n$ the relations $p\left(j, j^{\prime}, n\right)$ tell us precisely that $y_{n}$ is independent of the choice of $j \geqslant n$ for each $n$.

Let $U=R\left[W_{j}: j\right]$ and for each $n$ let $p_{n} \in U$ be defined by 


$$
p_{n}=y_{n}-\sum_{i=0}^{n} x_{i} W_{n-i}
$$

Let $J$ be the ideal of $U$ generated by the $p_{n}$ and let $S=U / J$.

We shall show that $R$ is pure in $S$ but that $R[[t]]$ is not cyclically pure in $S[[t]]$.

To see that $R$ is pure in $S$ let $S_{n}=R\left[W_{0}, \ldots, W_{n}\right] /\left(p_{0}, \ldots, p_{n}\right)$. Clearly, in an obvious way, $S=$ proj $\lim _{n} S_{n}$. Therefore, it suffices to show that $R \rightarrow S_{n}$ is pure for each $n$. But $R$ is a direct summand of $S_{n}$ as an $R$-module: in fact, $R$ is an $R$-algebra retract. To see this, simply map $R\left[W_{0}, \ldots, W_{n}\right] \rightarrow R$ (as $R$ algebras) by sending $W_{i}$ to $z_{i n}$. Then $p_{i}$ maps to $y_{i}-\sum_{i=0}^{n} x_{i} z_{n-i, n}=0,0 \leqslant i$ $\leqslant n$, and so we have the required $R$-algebra retraction

$$
S_{n}=R\left[W_{0}, \ldots, W_{n}\right] /\left(p_{0}, \ldots, p_{n}\right) \rightarrow R
$$

It remains to show that $R[[t]] \rightarrow S[[t]]$ is not cyclically pure. We simply exhibit a principal ideal of $R[[t]]$ which is not contracted. Let

$$
\begin{aligned}
& x=\sum_{i=0}^{\infty} x_{i} t^{i} \in R[[t]], \\
& y=\sum_{i=0}^{\infty} y_{i} t^{i} \in R[[t]]
\end{aligned}
$$

and

$$
w=\sum_{i=0}^{\infty} w_{i} t^{i} \in S[[t]]
$$

Then $y \in x S[[t]]$; in fact, the relations $p_{i}$ are precisely what we need to guarantee that $y=w x$. To complete the proof, it will suffice to show that $y \notin x R[[t]]$, for then $x R[[t]]$ is a principal ideal of $R[[t]]$ which is not contracted from $S[[t]]$.

Suppose, to the contrary, that $y \in x R[[t]]$. We shall derive a contradiction. The fact that $y \in x R[[t]]$ simply means that there are elements $r_{0}, \ldots, r_{i}, \ldots$ $\in R$ such that

$$
y=x \sum_{i=0}^{\infty} r_{i} t^{i},
$$

or, in other words, that the $r_{i}$ satisfy the countable system of equations 
(E)

$$
\left\{\begin{array}{c}
y_{0}=x_{0} r_{0}, \\
y_{1}=x_{1} r_{0}+x_{0} r_{1}, \\
\cdots \\
\cdots \\
\cdots \\
y_{n}=x_{n} r_{0}+\cdots+x_{0} r_{n}, \\
\cdots \\
\cdots \\
\cdots
\end{array}\right.
$$

Given a supposed solution, we can choose an integer $m$ so large that $r_{0} \in K\left[x_{0}, \ldots, x_{m}, z_{i j}, i \leqslant j \leqslant m\right]=R_{m} \subset R$. We shall obtain a contradiction by showing that if $r_{0} \in R_{m}$ then even the two equations

$\left(\mathrm{E}_{m}\right)$

$$
\left\{\begin{array}{c}
y_{m}=r_{0} x_{m}+\cdots+r_{m} x_{0}, \\
y_{m+1}=r_{0} x_{m+1}+\cdots+r_{m+1} x_{0},
\end{array}\right.
$$

have no solution in $R$.

To this end, we introduce an auxiliary ring. Let $U_{0}, U_{1}, V_{0}, V_{1}^{\prime}$, and $Q$ be indeterminates over $K$, let $B=K\left[U_{0}, U_{1}, V_{0}, Q\right]$, and let $A=B /\left(U_{0} Q\right)$. We denote the images of the variables in $A$ by $u_{0}, u_{1}, v_{0}, q$. We define $v_{0}^{\prime}=v_{0}+q$ and note that $u_{0} v_{0}=u_{0} v_{0}^{\prime}$.

Let $\phi$ be the unique $K$-homomorphism of $T=K\left[X_{i}, Z_{i j}: i \leqslant j\right]$ to $A$ such that:

$$
\begin{aligned}
\phi\left(X_{i}\right) & =0 \text { if } i<m \text { or } i>m+1, \\
\phi\left(X_{m}\right) & =u_{0} \text { and } \phi\left(X_{m+1}\right)=u_{1}, \phi\left(Z_{i j}\right)=0 \quad \text { if } i \geqslant 1, \\
\phi\left(Z_{0 j}\right) & =v_{0}, \quad \text { if } j \leqslant m, \\
\phi\left(Z_{0 j}\right) & =v_{0}^{\prime} \text { if } j \geqslant m+1 .
\end{aligned}
$$

It is easy to check that for all $n, j, j^{\prime}$, where $j, j^{\prime} \geqslant n, \phi\left(p\left(j, j^{\prime}, n\right)\right)=0$. Hence $\phi$ induces a $K$-homomorphism

$$
\psi: R \rightarrow A \text {. }
$$

Let $a_{i}$ denote the image of $r_{i}$ in $A$. Now, $\psi\left(R_{m}\right)=K\left[u_{0}, v_{0}\right] \subset A$ and so 
$a_{0} \in K\left[u_{0}, v_{0}\right]$. Moreover, $\psi\left(y_{\nu}\right)=\psi\left(\sum_{i=0}^{\nu} x_{i} z_{\nu-i, \nu}\right)$, and so $\psi\left(y_{\nu}\right)=0$ if $\nu$ $<m$ while $\psi\left(y_{m}\right)=u_{0} v_{0}$ and $\psi\left(y_{m+1}\right)=u_{1} v_{0}^{\prime}$. If we apply $\psi$ to the equations $\left(\mathrm{E}_{m}\right)$ we obtain

(1) $u_{0} v_{0}=u_{0} a_{0}$,

(2) $u_{1} v_{0}^{\prime}=u_{0} a_{1}+u_{1} a_{0}$,

over $A$, and we know $a_{0} \in K\left[u_{0}, v_{0}\right]$. Now, $U_{0} Q B \cap K\left[U_{0}, V_{0}\right] \subset Q B$ $\cap K\left[U_{0}, V_{0}\right]=0$, so that $K\left[u_{0}, v_{0}\right] \cong K\left[U_{0}, V_{0}\right]$. But then $u_{0} v_{0}=u_{0} a_{0}$ and $a_{0} \in K\left[u_{0}, v_{0}\right] \Rightarrow a_{0}=v_{0}$. Substituting in (2), we have $u_{1} v_{0}^{\prime}-u_{1} v_{0} \in u_{0} A$, or, passing to $B$, that $U_{1}\left(V_{0}+Q\right)-U_{1} V_{0} \in\left(U_{0}, U_{0} Q\right) B=U_{0} B$, whence $U_{1} Q \in U_{0} B$, a contradiction. Q.E.D.

\section{REFERENCES}

1. H. Bass, On the ubiquity of Gorenstein rings, Math. Z. 82 (1963), 8-28. MR 27 \#3669.

2. J. Brewer and D. Costa, Contracted ideals and purity for ring extensions, Univ. of Kansas, Lawrence and Univ. of Virginia, Charlottesville (preprint).

3. P. M. Cohn, On the free product of associative rings, Math. Z. 71 (1959), 380-398. MR 21 \#5648.

4. E. Enochs, On absolutely pure modules, Univ. of Kentucky, Lexington (preprint).

5. D. Ferrand and M. Raynaud, Fibres formelles d'un anneau local noethérien, Ann. Sci. École Norm. Sup. (4) 3 (1970), 295-311. MR 42 \#660.

6. R. Fossum, Commutative extensions by canonical modules and Gorenstein rings, Ȧrhus Univ. and Univ. of Illinois, Urbana (preprint).

7. H.-B. Foxby, Gorenstein modules and related modules, Math. Scand. 31 (1972), 267-284 (1973). MR 48 \#6094.

8. R. Gilmer, Contracted ideals in Krull domains, Duke Math. J. 37 (1970), 769-774. MR 42 \#3068.

9. R. Gilmer and J. Mott, Some results on contracted ideals, Duke Math. J. 37 (1970), 751-767. MR 42 \#3067.

10. A. Grothendieck (notes by R. Hartshorne), Local cohomology, Lecture Notes in Math., no. 41, Springer, New York, 1967. MR 37 \#219.

11. J. Herzog and E. Kunz, Der kanonische Modul eines Cohen-Macaulay-rings, Lecture Notes in Math., no. 238, Springer, New York, 1971.

12. M. Hochster, Contracted ideals from integral extensions of regular rings, Nagoya Math. J. 51 (1973), 25-43. MR 50 \#2149.

13. - Topics in the homological theory of modules over commutative rings, CBMS Regional Conf. Ser. in Math., no. 24, Amer. Math. Soc., Providence, R.I., 1975.

14. $M$. Hochster and J. Roberts, Rings of invariants of reductive groups acting on regular rings are Cohen-Macaulay, Advances in Math. 13 (1974), 115-175. MR 50, 311.

15. - The purity of the Frobenius and local cohomology, Advances in Math. 21 (1976), $117-172$.

16. I. Kaplansky, Commutative rings, Allyn and Bacon, Boston, 1970. MR 40 \# 7234.

17. E. Matlis, Injective modules over Noetherian rings, Pacific J. Math. 8 (1958), 511-528. MR 20 \#5800.

18. H. Matsumura, Commutative algebra, Benjamin, New York, 1970. MR 42 \#1813.

19. I. Reiten, The converse to a theorem of Sharp on Gorenstein modules, Proc. Amer. Math. Soc. 32 (1972), 417-420. MR 45 \#5128.

20. J. Rotman, Notes on homological algebra, Van Nostrand Reinhold Math. Studies, no. 26, Van Nostrand Reinhold, New York, 1970. 
21. J.-P. Serre, Algèbre locale. Multiplicités. Lecture Notes in Math., no. 11, Springer, New York, 1965. MR 34 \# 1352.

22. R. Y. Sharp, Gorenstein modules, Math. Z. 115 (1970), 117-139. MR 41 \#8401.

23. - On Gorenstein modules over a complete Cohen-Macaulay local ring, Quart. J. Math. Oxford Ser. (2) 22 (1971), 425-434. MR 44 \#6693.

24. R. B. Warfield, Jr., Purity and algebraic compactness for modules, Pacific J. Math. 28 (1969), 699-719. MR 39 \#4212.

Department of Mathematics, Purdue University, West Lafayette, Indiana 47907

Current address: Department of Mathematics, University of Michigan, Ann Arbor, Michigan 48109 